Bio - grafia. Escritos sobre la Biología y su Enseñanza. ISSN 2027

Edición Extraordinaria. p.p. $1781-1790$

Memorias del VIII Encuentro Nacional de Experiencias en Enseñanza de la Biología y la Educación Ambiental. III Congreso Nacional de Investigación en Enseñanza de la Biología.

\title{
EL PAPEL DEL TRABAJO COLABORATIVO DENTRO DEL ENFOQUE INVESTIGACIÓN DIRIGIDA EN LA ENSEÑANZA DE ESTADOS DE LA MATERIA: UNA EXPERIENCIA EN GRADO QUINTO DE PRIMARIA.
}

\section{THE ROLE OF COLLABORATIVE WORK WITHIN THE APPROACH RESEARCH DIRECTED IN THE TEACHING OF STATES OF MATTER: AN EXPERIENCE IN FIFTH GRADE}

B

Palabras clave: trabajo colaborativo, Investigación Dirigida, Unidad Didáctica, cambios actitudinales, prácticas.

Abstract.

This study aims to determine the effectiveness of collaborative work, their role and relevance within the didactic approach Research Directed by teaching the concept of states of matter. For it was designed and implemented a teaching unit called "Young Scientists 5: Inquiring about the states of matter in nature" six class sessions in Venice Cooperative College, in the fifth grade. The practical proposals were analyzed by students to conceptual, procedural and attitudinal level to demonstrate the effect of collaborative work from four basic aspects: 
Bio - grafia. Escritos sobre la Biología y su Enseñanza. ISSN 2027

Edición Extraordinaria. p.p. 1781-1790

Memorias del VIII Encuentro Nacional de Experiencias en Enseñanza de la Biología y la Educación Ambiental. III Congreso Nacional de Investigación en Enseñanza de la Biología.

Participation and shared responsibility, freedom of expression, autonomy and self-regulation; in teaching states of matter.

Keywords: collaborative work, Directed Research, Teaching Unit, attitudinal changes, practice.

\section{INTRODUCCIÓN}

Este estudio tiene como objetivo determinar la eficacia del trabajo colaborativo, su papel y relevancia dentro del enfoque didáctico Investigación Dirigida, mediante la construcción del concepto de estados de la materia, en estudiantes de grado quinto de primaria.

Dentro del eje de la enseñanza y el aprendizaje de las ciencias, es importante explorar los medios de investigación en el aula oportunos para abordar el ambiente escolar dentro del enfoque de Investigación Dirigida propuesto por Pozo y Gómez (2004).

Tal como lo define Zamora (2005), la investigación dirigida es una actividad experimental que requiere la participación activa del estudiante y que orienta la búsqueda de una evidencia que permita resolver un problema práctico o contestar un cuestionamiento teórico, motivando cambios actitudinales, procedimentales y conceptuales en los estudiantes, acercándolos al conocimiento científico (Cañal y Porlán, 1987).

En la Investigación Dirigida, además de los conceptos y los procedimientos, es preciso identificar las variables que se tomarán en consideración, ya que esto permitirá orientar a los niños en el proceso experimental, y les facilitará el camino para encontrar respuesta a los problemas planteados (Zamora, 2005). En este sentido, el trabajo colaborativo permite en el aula de clase el desarrollo de habilidades individuales y grupales a partir de la discusión entre los estudiantes de un concepto nuevo, buscando que cada uno sea responsable de su propio aprendizaje (EAFIT, 2014).

El aprendizaje colaborativo desarrollado en el aula genera el intercambio de conceptos e ideas, para así, desarrollar habilidades comunicativas y sociales que de indicio de una reestructuración cognitiva entre los estudiantes lo que permite la resolución de problemas entre los miembros de un grupo de trabajo, trascendiendo a la teoría planteada por Vygotsky de la "zona del desarrollo próximo" (EAFIT, 2014) 
Bio - grafia. Escritos sobre la Biología y su Enseñanza. ISSN 2027

Edición Extraordinaria. p.p. 1781-1790

Memorias del VIII Encuentro Nacional de Experiencias en Enseñanza de la Biología y la Educación Ambiental. III Congreso Nacional de Investigación en Enseñanza de la Biología.

Desde el aprendizaje colaborativo se tomaron unas condiciones fundamentales para el trabajo colaborativo: Participación y responsabilidad compartida; en donde los integrantes del grupo comparten la responsabilidad del trabajo de las guías; Libertad de expresión: Se genera un clima en el grupo de respeto a las opiniones de cada integrante; Capacidad de Dialogo: Los miembros del grupo deben discutir activamente y expresar opiniones que generen una solución a un problema; Autorregulación y autonomía: Los grupos deben estar atentos a los procesos generados en la solución de los procedimientos y así poder discutir los objetivos y procedimientos, se propensa a aplicar y examinar las experiencias desarrolladas (EAFIT, 2014)

\section{METODOLOGÍA}

Se diseñó e implementó la Unidad Didáctica: "Pequeños científicos 5: Indagando acerca de los estados de la materia en la naturaleza", para seis sesiones de clase, en el grado quinto de primaria del Colegio Cooperativo Venecia- Bogotá, D.C. En la última sesión, los estudiantes desarrollaron una guía de laboratorio final, en donde explicaron los elementos que tenía que poseer su práctica final (como la pregunta de investigación, la metodología a emplear, los resultados y la discusión de los mismos). Finalmente se realizó una socialización en la que cada grupo de trabajo expuso los resultados de su montaje final y dieron cuenta de la resolución de la pregunta de investigación.

A partir de los resultados arrojados por las guías proporcionadas, la caracterización de ideas previas; así como la documentación y la evidencia filmográfica, se procedió a evidenciar la trascendencia del trabajo colaborativo. Para ello se tuvo en cuenta el Diario del profesor (Porlán \& Martín, 2004), la observación participante y el análisis documental (Ortega, 2008). La metodología anterior, se sustenta desde una mirada interpretativa-hermenéutica, que busca la comprensión de los fenómenos (Kuhn, 1970).

\section{RESULTADOS Y ANÁLISIS}

Se tomo un grupo focal (Mella, 2008). El análisis consistió en visibilizar la construcción grupal del montaje final para evidenciar el efecto del trabajo colaborativo en la Investigación dirigida. 
Bio - grafia. Escritos sobre la Biología y su Enseñanza. ISSN 2027

Edición Extraordinaria. p.p. 1781-1790

Memorias del VIII Encuentro Nacional de Experiencias en Enseñanza de la Biología y la Educación Ambiental. III Congreso Nacional de Investigación en Enseñanza de la Biología.

En la Tabla 1 se muestra el montaje desarrollado por el grupo 1, Modelaron la pregunta de investigación hacia un sentido más microscópico, al cuestionarse sobre las reacciones acaecidas que se presentan mediante la mezcla de elementos.

\begin{tabular}{|c|c|c|}
\hline \multicolumn{3}{|c|}{ GRUPO 1} \\
\hline \multirow[t]{2}{*}{ Pregunta de investigación. } & Primera sesión & \\
\hline & $\begin{array}{llr}\text { "¿Qué pasa } & \text { si } \\
\text { mezclamos } & \text { hielo } & y \\
\text { Dióxido } & & d e \\
\text { Carbono?" } & & \end{array}$ & $\begin{array}{l}\text { "¿Cómo se presentan las } \\
\text { reacciones químicas?" }\end{array}$ \\
\hline Objetivos de la investigación. & \multicolumn{2}{|c|}{$\begin{array}{l}\text { 1. "Porque al mezclar bicarbonato y vinagre se } \\
\text { puede crear } \mathrm{CO}_{2} \text { ?" } \\
\text { 2. "En el experimento queremos verificar } \\
\text { cambios físicos y químicos cuando el agua se } \\
\text { expone al } \mathrm{CO}_{2 . "}\end{array}$} \\
\hline Hipótesis. & \multicolumn{2}{|c|}{$\begin{array}{l}\text { "Podría ser que el hielo al exponerse al } \mathrm{CO}_{2} \text { puede } \\
\text { que se derritan sus moléculas o puede que no." }\end{array}$} \\
\hline Procedimiento/Metodología & \multicolumn{2}{|c|}{$\begin{array}{l}\text { "Materiales: } 4 \text { platos, sal, azúcar, bicarbonato de } \\
\text { sodio, vinagre, hielos. } \\
\text { 1. Marcar los } 4 \text { platos con } 1,2,3,4 \text {. } \\
\text { 2. Colocar los hielos en cada plato. } \\
\text { 3. Colocar el primer plato y el azúcar, en el } \\
\text { segundo plato sal, tercer plato limón, y en el } \\
\text { cuarto nada. } \\
\text { 4. Luego agregamos bicarbonato a todos los } \\
\text { platos. } \\
\text { 5. Agregamos vinagre a todos los platos. } \\
\text { 6. Observar cuales cambios suceden en cada } \\
\text { plato." }\end{array}$} \\
\hline
\end{tabular}


Bio - grafia. Escritos sobre la Biología y su Enseñanza. ISSN 2027

Edición Extraordinaria. p.p. 1781-1790

Memorias del VIII Encuentro Nacional de Experiencias en Enseñanza de la Biología y la Educación Ambiental. III Congreso Nacional de Investigación en Enseñanza de la Biología.

\begin{tabular}{|l|l|}
\hline Resultados y Análisis. & "Que al mezclar dos sustancias químicas y una física \\
se derritió más rápido el hielo 3 del plato 3. \\
El tercer hielo se derritió más rápido, el segundo se \\
derritió de segundas, el primero de terceras, y el \\
cuarto de cuartas."
\end{tabular}

Tabla 1. Aspectos desarrollados en el montaje propuesto por el grupo 1.

A partir de lo consignado en el diario de campo, durante el seguimiento dado al grupo, se identificó que la asignación de funciones de trabajo se llevo a cabo en las sesiones realizadas. Cada estudiante cumplía a cabalidad sus funciones asignadas desde la primera sesión, hasta la última. Los integrantes desarrollaron un montaje donde se pudiera diferenciar los cambios físicos y químicos de la materia para lo cual utilizaron un cubo de hielo para frotar tiras de papel hasta que estuvieran totalmente húmedas observando un cambio físico; y posteriormente acercaron a un mechero para observar los cambios químicos que se presentaban en el papel.

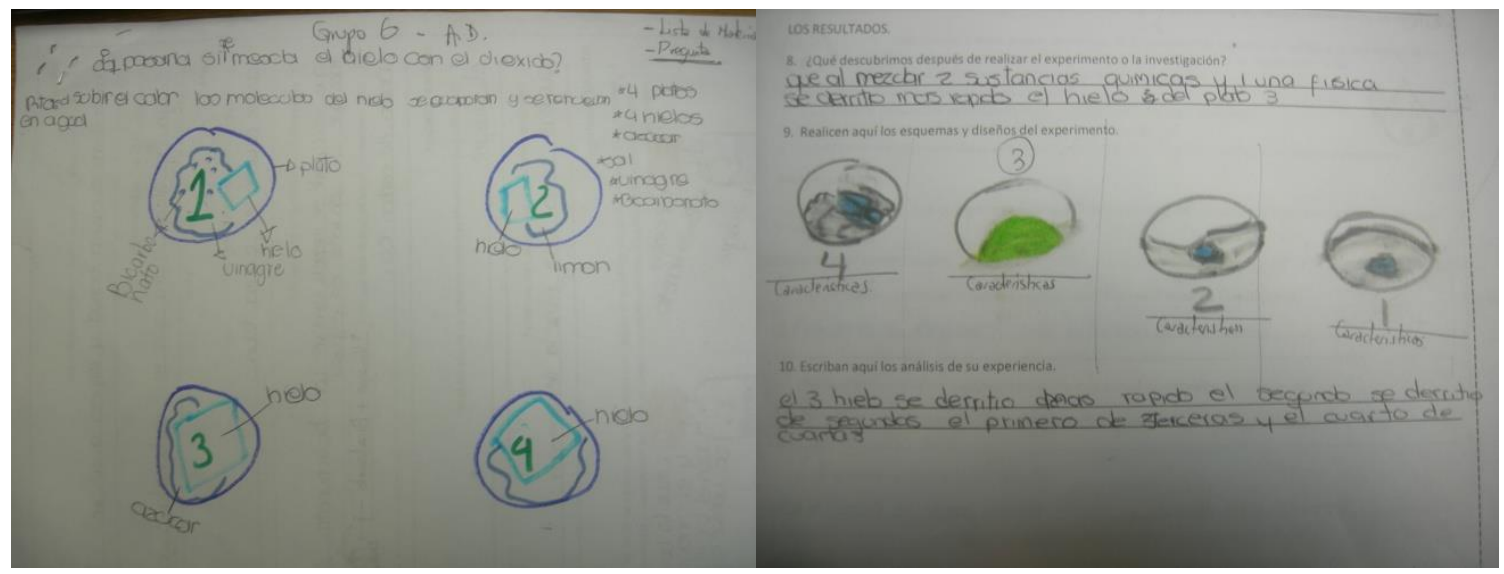

Imagen 1 y 2: Montaje realizado por el grupo 1.

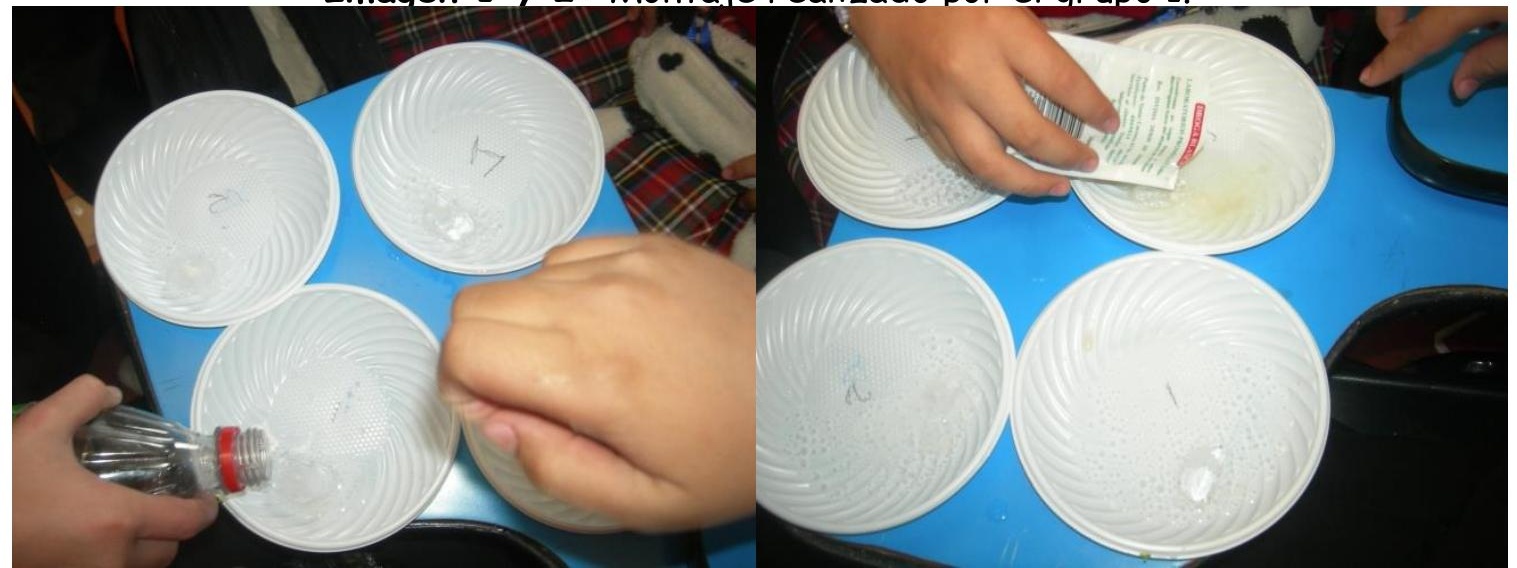


Bio - grafia. Escritos sobre la Biología y su Enseñanza. ISSN 2027

Edición Extraordinaria. p.p. 1781-1790

Memorias del VIII Encuentro Nacional de Experiencias en Enseñanza de la Biología y la Educación Ambiental. III Congreso Nacional de Investigación en Enseñanza de la Biología.

Imagen 3 y 4: Elaboración experimento del grupo 1.

De las prácticas realizadas con los grupos de trabajo, se realizó una retroalimentación en el aula para explicar los conceptos que se habían apropiado, cómo se llevo a cabo el proceso de construcción del montaje propuesto y los medios que utilizaron para explicar el concepto dado. En la siguiente tabla se evidencia el aprendizaje conceptual, procedimental y actitudinal que presentaron los estudiantes hasta la última sesión.

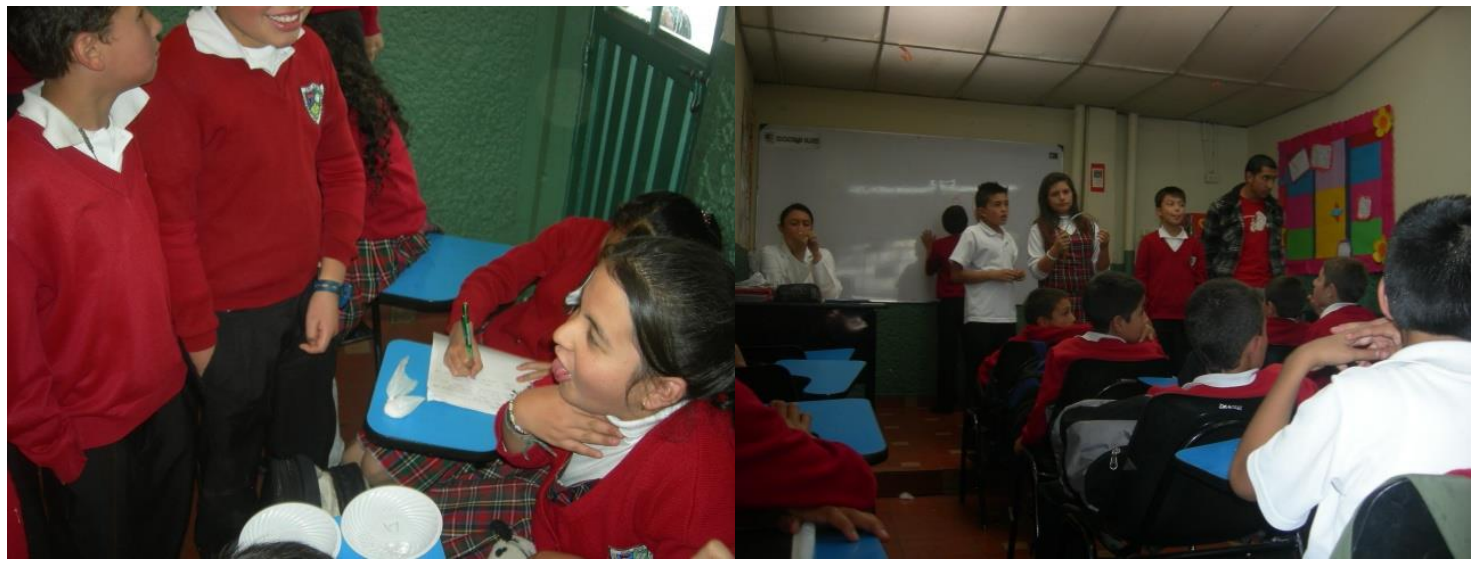

Imagen 5 y 6: Discusión en el grupo y socialización.

\begin{tabular}{|l|l|}
\hline GRUPO & Aprendizaje motivado por el trabajo colaborativo. \\
\hline
\end{tabular}


Bio - grafia. Escritos sobre la Biología y su Enseñanza. ISSN 2027

Edición Extraordinaria. p.p. $1781-1790$

Memorias del VIII Encuentro Nacional de Experiencias en Enseñanza de la Biología y la Educación Ambiental. III Congreso Nacional de Investigación en Enseñanza de la Biología.

\begin{tabular}{|c|c|c|c|}
\hline & $\begin{array}{l}\text { Aprendizaje } \\
\text { Conceptual }\end{array}$ & $\begin{array}{l}\text { Aprendizaje } \\
\text { Procedimental }\end{array}$ & Aprendizaje Actitudinal \\
\hline 1 & $\begin{array}{l}\text { Cuando hacemos } \\
\text { reaccionar dos } \\
\text { materiales, nos va a } \\
\text { dar origen a otro } \\
\text { nuevo. Por ejemplo: } \\
\text { al mezclar } \\
\text { bicarbonato de } \\
\text { sodio con vinagre } \\
\text { nos va a dar Dióxido } \\
\text { de Carbono. Esto es } \\
\text { un cambio químico y } \\
\text { da como resultado } \\
\text { una reacción } \\
\text { química. }\end{array}$ & $\begin{array}{l}\text { No se debe comer en } \\
\text { el salón o el } \\
\text { laboratorio. } \\
\text { No tocar nada. } \\
\text { No hacer nada hasta } \\
\text { que los docentes lo } \\
\text { indiquen. } \\
\text { Utilizar bata de } \\
\text { laboratorio. }\end{array}$ & $\begin{array}{l}\text { Escuchar antes de hablar. } \\
\text { Respetar la palabra del } \\
\text { compañero. } \\
\text { Respetar a los compañeros. } \\
\text { El trabajo en grupo nos } \\
\text { sirve porque nos podemos } \\
\text { apoyar. } \\
\text { Hay que hacerle caso al } \\
\text { profesor porque hay que } \\
\text { respetarlo. }\end{array}$ \\
\hline
\end{tabular}

Tabla 3. Cambios en el aprendizaje motivados por el trabajo colaborativo.

A partir del esquema anterior es preciso determinar que, a partir del trabajo colaborativo, se generó una interdependencia positiva, generada al interior del equipo de trabajo, obteniendo la consecución de una meta común (EAFIT, 2014). Esto ayuda a elevar el autoestima y la confianza de los estudiantes, para trabajar en equipo al emplear el enfoque didáctico Investigación Dirigida.

La primera condición evaluada fue la participación y responsabilidad compartida que se desarrolló desde la primera clase hasta finalizar, en la primera guía proporcionada hubo un énfasis en esta categoría; los grupos debían formarse de manera que los estudiantes desarrollaran preguntas tales como: ¿Qué normas se deben llevar a cabo en el laboratorio?, donde se evaluó el respeto al profesor y a los integrantes del grupo. 
Bio - grafia. Escritos sobre la Biología y su Enseñanza. ISSN 2027

Edición Extraordinaria. p.p. 1781-1790

Memorias del VIII Encuentro Nacional de Experiencias en Enseñanza de la Biología y la Educación Ambiental. III Congreso Nacional de Investigación en Enseñanza de la Biología.

Desde la Libertad de expresión y a la capacidad de diálogo, los estudiantes tuvieron la oportunidad de generar respuestas espontáneas frente a la elaboración de su experimento final, proporcionando un análisis que surgía desde la discusión permanente del fenómeno que se evidenciaba en el experimento, reforzado por un liderazgo permanente llevado a cabo por dos estudiantes, cuya comunicación facilitada contribuía al buen desarrollo de las actividades. Una afirmación importante, como aporte a la investigación fue la que concedió un estudiante que pertenecía a este grupo de trabajo: "Lo que observé fue que al mezclar dos sustancias, como el bicarbonato y el vinagre se genera una especie de cambio, donde nace otra sustancia "nueva", así como se forman los compuestos". En lo anterior, cabe destacar la definición microscópica que aporta el estudiante frente a lo que observa, lo que ayuda a construir un análisis de resultados que surge desde la investigación científica (Pozo \& Gómez, 2004). 
Bio - grafia. Escritos sobre la Biología y su Enseñanza. ISSN 2027

Edición Extraordinaria. p.p. 1769-1780

Memorias del VIII Encuentro Nacional de Experiencias en Enseñanza de la Biología y la Educación Ambiental. III Congreso Nacional de Investigación en Enseñanza de la Biología.

En cuanto a la autorregulación, el procedimiento que se logra a través de los objetivos planteados muestra una clara interpretación microscópica de los estados de la materia. Sin embargo, llevar a cabo la metodología establecida por estudiantes de quinto grado no es fácil, evidenciando que de alguna manera los estudiantes no tienen alguna cercanía al método científico, por lo que se les dificultaba plantear un objetivo. Esta problemática se agudiza desde el momento en que a los estudiantes se les proporciona la autonomía suficiente para determinar sus propias reglas de juego, atribuído a su estadío de desarrollo moral heterónomo.

El contenido realizado por el grupo 1 fue abordado a partir de los cambios que se presentan en los diferentes estados de la materia, poniendo énfasis en las reacciones químicas de un elemento vital como es el agua. Es aquí donde se evidencia una relación con el contexto, permitiendo que los estudiantes aplicaran el concepto a la realidad donde viven. El trabajo colaborativo propicia que el aprendizaje conceptual de los estados de la materia trascienda del aula de clase, alcanzando un aprendizaje actitudinal, un punto que propone Cañal \& Porlán (1987) (Cfr: EAFIT, 2014).

\section{CONCLUSIONES}

La investigación Dirigida puede ser un método usado por los profesores de ciencias para acercar a los estudiantes a la realidad de un científico, sin pretender que esta sea la realidad definitiva. Un punto importante de este enfoque didáctico es la importancia del trabajo colaborativo, mediante la conformación de grupos de trabajo pequeños, incentivando el acercamiento del conocimiento científico a la realidad cotidiana.

El trabajo colaborativo dentro del concepto de estados de la materia, contribuye a la construcción de este, mediante el debate crítico y la socialización de métodos para determinar los cambios que suceden en la materia. Desde el punto de vista educativo, la materia es un componente de estudio importante, que debe reforzarse en la educación secundaria.

Desde los cuatro aspectos revisados, se determina que el papel del trabajo colaborativo es relevante, siempre y cuando el docente implemente prácticas que incentiven los trabajos grupales, que muestran la construcción conceptual mediante la interacción con los otros 
Bio - grafia. Escritos sobre la Biología y su Enseñanza. ISSN 2027

Edición Extraordinaria. p.p. 1769-1780

Memorias del VIII Encuentro Nacional de Experiencias en Enseñanza de la Biología y la Educación Ambiental. III Congreso Nacional de Investigación en Enseñanza de la Biología.

partícipes de la enseñanza. No hay que recaer en el discurso individualista que siempre ha tenido la indagación científica, un obstáculo que se puede superar mediante la implementación del enfoque didáctico Investigación dirigida. El trabajo colaborativo refuerza el propósito de enseñanza de este enfoque propuesto por Pozo y Gómez, cuyos sujetos siempre están prestos a la contestación permanente de preguntas que nacen desde la evidencia empírica y desde el acompañamiento permanente del profesor de ciencias.

\section{REFERENCIAS BIBLIOGRÁFICAS.}

Cañal, R., \& Porlán. (1987). Investigando la realidad próxima: Un modelo didáctico alternativo. Enseñanza de las ciencias.

EAFIT, U. (14 de Noviembre de 2014). Aprendizaje Colaborativo/Cooperativo. Obtenido de Colombia Aprende:

<http://www.colombiaaprende.edu.co/html/mediateca/1607/articles167925_archivo.pdf

Kuhn, T. (1970). Estructura de las revoluciones científicas. Londres: University of Chicago press.

MARSHALL, C., \& ROSSMAN, G. (1995). Designing qualitative research. . KAWULICH, B. (2005). La observación participante como método de recolección de datos. Forum Qualitative social research.

Mella, O. (2008). Grupos Focales "Focus Groups" Técnica de Investigación Cualitativa. CIDE.

Ortega, L. (2008). El diseño metodológico en un proyecto de investigación socioeducativa. EDUCACIÓN SUPERIOR: Revista Pedagógica Nacional.

Porlán, R., \& Martín, J. (2004). El Diario del profesor: un recurso para la investigación en el aula. Sevilla: Editorial Díada.

Pozo, J. I., \& M.A., G. C. (2004). Aprender y Enseñar ciencia. Madrid: Editorial Morata. 
Bio - grafia. Escritos sobre la Biología y su Enseñanza. ISSN 2027

Edición Extraordinaria. p.p. $1769-1780$

Memorias del VIII Encuentro Nacional de Experiencias en Enseñanza de la Biología y la Educación Ambiental. III Congreso Nacional de Investigación en Enseñanza de la Biología.

Pozo, J., \& Gómez, M. (2004). Enseñando a comprender la Naturaleza de la Materia: el dialogo entre la química y nuestros sentidos. Educación química 15.

Zamora, A. (. (2005). La Investigación Dirigida. Congreso Nacional de Ciencias. Exploraciones fuera del aula. Costa Rica. 